\title{
AN INTELLIGENT CHINESE PHONETIC ON-SCREEN VIRTUAL KEYBOARD SYSTEM
}

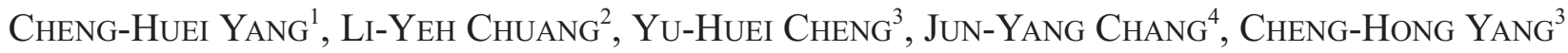 \\ ${ }^{1}$ Department of Electronic Communication Engineering, \\ National Kaohsiung Marine University, \\ ${ }^{2}$ Department of Chemical Engineering, I-Shou University, \\ ${ }^{3}$ Department of Electronic Engineering, \\ National Kaohsiung University of Applied Sciences, \\ ${ }^{4}$ Department of Information Management, \\ National Kaohsiung University of Applied Sciences, Kaohsiung, Taiwan
}

\begin{abstract}
In this paper, we designed and implemented a user-friendly Chinese phonetic on-screen virtual keyboard key-in system for persons with disabilities. The proposed system inputs the Chinese characters by way of the mouse selecting phonetic symbols. After selecting the initial phonetic symbol, a list of possible phonetic symbol combinations is shown. The proposed system can decrease the number of input clicks needed to select a character, thereby improving typing speed. In addition, the size of the keyboard can be adjusted to increase accuracy and convenience. The system also provides temporary saving and scanning of selections, which makes the keyboard convenient for users. The most frequently typed characters typed by an individual user will be ranked first and this ranking constantly adjusted to facilitate character selection. Moreover, the proposed system shows common combinations of the character typed with other Chinese characters. The system will list all of the possible term correlations for a user to choose from, so that the user spends considerably less time inputting frequently used character combinations. Experimental results showed that the proposed on-screen virtual keyboard system provides an operation interface, which is easier to use, and achieves faster typing speeds compared to two other systems tested.
\end{abstract}

Biomed Eng Appl Basis Comm, 2006(August); 18: 178-184.

Keywords: morse code; chinese phonetic system; keyboard.

\section{INTRODUCTION}

Received: Feb. 14, 2006; Accepted: May 29, 2006

Correspondence: Cheng-Hong Yang, Professor

Department of Electronic Engineering, National

Kaohsiung University of Applied Sciences, Kaohsiung,

Taiwan

E-mail: chyang@cc.kuas.edu.tw
Rapid technological development takes advantage of information and communication technology to assist the improvement of our living environment. Computer hardware and software functions not only support teaching or learning, but also provide vocational and recreational tools used in everyday life. In order to improve the quality of life for persons with disabilities in our knowledge-based society and to let them make contributions to the technology-based economic system in the future, they have to be able to fully participate in an information-learning environment. 
Besides establishing unimpeded access to network environments, decreasing the difficulties of computer manipulation is an important objective. Many auxiliary computer tools have been developed to assist the disabled, such as key guards, guard trackballs, mouse substitution equipment such as joysticks, alternate keyboards (programmable keyboards, miniature keyboards, expanded keyboards, on-screen keyboards, etc.), single-key switches for input by Morse Code, selection scanning, voice recognition, handwriting recognition, and optical character recognition (OCR) with a scanner [1-4].

Chinese computer usage has been fully developed, but it is limited by the Chinese input method. Through efforts by people with various experiences, different Chinese input methods have been developed. Examples are the Triangle input method by Huang Ke-tung, the Chang Jie input method by $\mathrm{Chu}$ Bang-Fu, the Head and End numbers input method by Chen Shuen-Chi, the Da Yi input method by Chi Tung-Chieh, the BosShiAmy input method by Liu Chung-Tz, the Wang Code input method by Wang Yiao-Shi, and the Wah-Chiang input method by Chen Wah-Wei. Even though different design concepts have lead to different input methods, each method has the same objective, i.e. it must be "easy to learn," " fast," and "unforgettable [5]."

Luo and his coworkers developed a Chinese Morse code using the Chinese Phonetic input method to help some disabled people use a Chinese computer [6]. Luo's Chinese phonetic Morse code is characterized by a prefix style, which makes it difficult to memorize. Yang developed a new Chinese phonetic Morse code, which is based on three aspects: the appearance frequency of prefixes, the writing order of strokes, and the shapes of characters. Experimental results showed that the new Morse code is simpler, faster, and easier to remember than Luo's method. Additionally, it saves typing time. This new Morse code is highly efficient in assisting disabled people who want to use a Chinese computer.

According to the computer skill test results conducted by the Chinese Computer Foundation of the Republic of China, one commonly Chinese input method is the phonetic symbol input method (Bo Po Mo Fo). This input method is easy to learn and use [6]. It uses 37 symbols and 5 tones to represent the possible Mandarin sounds. The phonetic symbols are entered initially and then a list of corresponding Chinese characters is provided for input selection. Aside from providing a suitable keyboard input surface, a reliable Chinese input method is strictly required. In previous literature reports, the Chinese character input methods were divided into four kinds. The first kind inputs the Chinese character using a common keyboard based on the Chinese input method of the operating system. The second kind uses auxiliary keyboards that are also based on the Chinese input method of the operating system, such as a miniature keyboard, a wisdom keyboard, or a keyboard style mouse etc. [8]. The third kind is an input method in which handwritten or audio samples can be recognized. The last kind uses a movable cursor to select phonetic symbols on a virtual keyboard on the computer screen. The on-screen keyboard is often relatively small, making it often difficult for persons with physical impairments to correctly select the desired symbols, and therefore resulting in numerous erroneous entries. The Chinese input method that commonly ships with the operating system is also not convenient in these cases, since their require frequent mouse movements.

In this paper, we introduce a virtual on-screen keyboard that is easy to use for the persons with disabilities, since it requires fewer input clicks than comparable keyboards. The system facilitates simple computer tasks like sending and receiving emails browsing the Internet, or typing and editing data for users with physical impairments. The proposed software uses a mouse cursor to input characters and provides phonetic symbols and numerals, as well as symbol input functions. Once the first phonetic symbol is selected, a line with all possible phonetic combinations for the first phonetic symbol is shown, thus erroneous entries are far fewer than if the user would have to choose an entry from the remaining 36 symbols, and typing speed can be improved considerably. Furthermore, the size of keyboard can be adjusted to increase accuracy. The system provides a temporary holding and scanning function to facilitate use of the keyboard. A user only needs to move the mouse cursor over his intended selection and then the system will automatically select this symbol after the cursor is held there for a preset time. When a user selects the scanning option, the online keyboard will visually highlight a row where the intended symbol is located, and the slowly pan over the keys of that row. Once the desired key is highlighted is can be chosen by simply clicking with the mouse.

The Chinese characters for a given combination of phonetic symbols are listed in descending order, with the most commonly used character listed first. This can considerably increase the typing speed. Furthermore, for any entered Chinese character a list with other often used combinations containing this character is shown, so that a second Chinese character making up a Chinese term can directly be chosen from that list of character related combinations, and does not have to be input separately. This again cuts the number of necessary keystrokes considerably. Experimental results have shown that the proposed software facilitates the input of Chinese and English characters and/or symbols for a general user, and is especially 
useful for users with physical impairments. It supports various types of mouse devices, such as the mouth control mouse and the head control mouse.

\section{SYSTEM DESIGN}

Currently, there are three kinds of miniature onscreen keyboards:

(1) Traditional on-screen miniature keyboard: based on the traditional phonetic symbol input method: provides a simple phonetic alphabetical keyboard, as well as a symbol keyboard for user to selection.

(2) Auxiliary on-screen miniature keyboard provided by Microsoft [9]: based on the exchange of input methods of the operating system; inputs characters that are similar to regular keyboard, also provides a temporary holding and scanning function.

(3) Click-N-Type keyboard with additional functions (Fig. 1 and Fig. 2): Click-N-Type keyboards can act on fields, such as the "email address", the "receiver" and "subject" row etc [10]. They also provide auto click and scanning functions, a character arrangement mode, as well as macros, which can input the preset words at a given time.

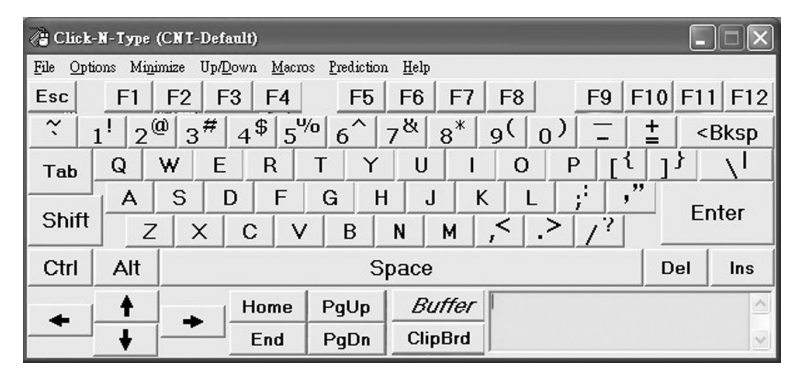

Fig. 1. English Click-N-Type keyboard.

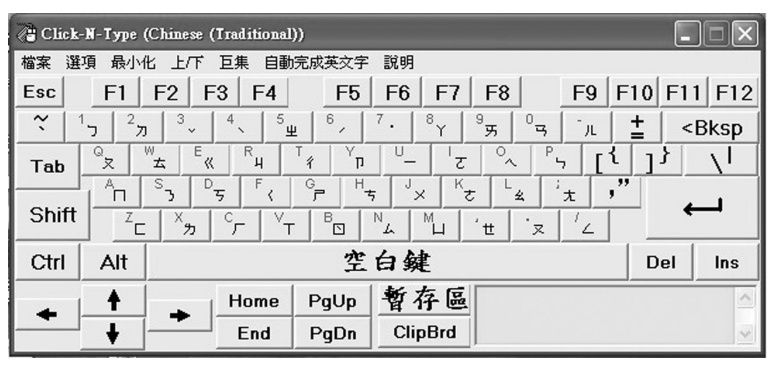

Fig. 2. Chinese Click-N-Type keyboard .
Our proposed keyboard contains six major functions:

1. File: Can open new and old files, save files, or close them.

2. Keyboard: provides for input of the English alphabet, numbers, special symbol input, which is subdivided into punctuation marks and mathematical symbols, the Chinese phonetic alphabet, measure units, the Greek alphabet, and other specialized marks, depending on the user's choice. The standard keyboard doesn't contain any special keys, for Print Screen, Insert, Delete, Page up, and Page Down functions. The enhanced keyboard setting includes these special keys on the keyboard.

3. Size: here the keyboard size can be adjusted. Enlarging the keyboard size can considerable improve the accuracy of the user's clicks. Shrinking the keyboard size can enlarge the video space on the screen when necessary. Using a single key as a measure unit, the size of the keyboard can be set to small $(30 * 20)$, medium $(35 * 25)$, large $(40 * 30)$, and ultra large-scale $(50 * 35)$. The system also provides an automatic size management function, which a user can apply to automatically swap window and keyboard sizes if required.

4. Options: this section is divided into the following five sub-categories:

(1) The keyboard can be set to stay on top of all other application windows.

(2) Sound when a mouse click results in a key selection.

(3) The input mode is divided into three kinds: one stroke selection, temporary holding selection, and scanning selection. These subfunctions are described below in detail:

(a) One stroke selection: As soon as the mouse hovers over a key it is highlighted in green. A simple click with the mouse activated this key.

(b) Temporary holding selection: The mouse cursor is moved over the key to be selected, which will automatically be chosen after a preset time the cursor spends over this key. The temporary delay time is defined as the time between holding and selecting a key and the next selection of a key. A repeat temporary holding selection can be set if the temporary holding selection will be used again.

(c) Scanning selection: The mouse cursor moved over a given row of keys on the keyboard will activate a scanning mechanism, in which the keys in that row are individually highlighted in green for a short time until the following key is highlighted. Once the cursor is over the key 
to be selected, a simple mouse click activates this key and its color turns to red once activated. Keys can be scanned forwards or backwards in a given row.

(4) Word editor panel (Fig. 3): here words that were input before can be edited individually before sending them to an application.

(5) The quick toolbar provides fast access for a user to software applications most commonly used on the system. This toolbar can be customized by the user, allowing him to set pathways to his preferred software applications.

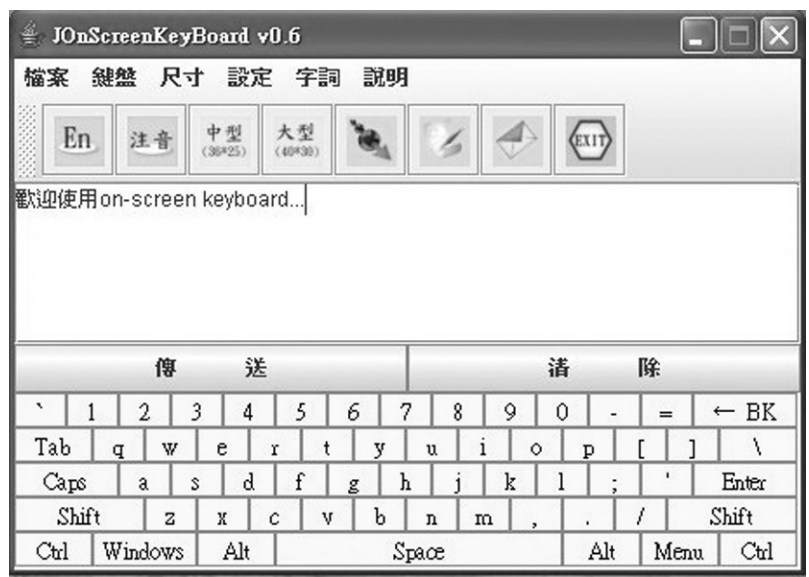

Fig. 3. The word editor panel.

5. Commonly used words: Provides a list with the most commonly words used in order of their frequency.

(a) Commonly typed words can be sorted in the order in which they were entered.

(b) Words can also be sorted according to the frequency with which they are used.

(c) The typed words can be converted to a text file. The contents of this text file can then be scanned for words of high priority.

(d) The user can create his own reference list with words he frequently uses in the text.

6. Help: contains a section with the version and credits for this software, and an introduction to the help topics.

When using the Chinese phonetic symbols in order to input Chinese characters, the proposed onscreen keyboard has a great advantage over currently available on-screen keyboards. As mentioned earlier, there are 37 Chinese symbols. As soon as the first one is entered, a new row appears on the keyboard showing all the possible combinations of phonetic symbols with the first one entered. This row contains considerably fewer keys then the 36 options to choose from on a conventional keyboard, since certain combinations of
Chinese phonetic symbols are not allowed. In Fig. 4 the symbol` was entered. The new row shows that this symbol can only be combined with 11 other symbols to make a Chinese sound. The 25 symbols that can not be phonetically combined with the symbol \ are not shown, thus making selection a lot easier, as well as preventing typing errors. Once the second phonetic symbol is added to the symbol 3 , and one of the 4 Chinese tones or the neutral tone is marked, all Chinese characters having that sound will appear on the bottom row, and can be chosen with a mouse click.

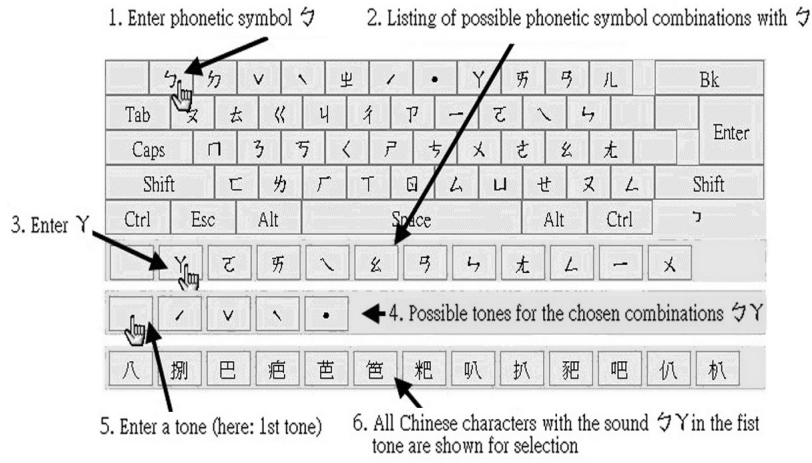

Fig. 4. Chinese phonetic keyboard showing the extra row with allowed phonetic symbol combinations.

\section{RESUTLS AND DISCUSSIONS}

A good Chinese phonetic symbol on-screen virtual keyboard is desperately needed, especially since many everyday users are not very familiar with the phonetic symbols. Some people have difficulties distinguishing certain phonetic symbol, for example “又” and “ट”, and “ฯ” and “世”, because they sound very familiar. Often a user types either “又” or “ट” after the phonetic symbol刀. Actually, no “ट" is phonetically allowed after typing ग, so an additional row on the keyboard showing all possible combinations is a great benefit, since these erroneous entries can be omitted.

The system is implemented using Java, Windows version. The proposed keyboard software supplies the following characteristic in order to provide a person with disabilities with an on-screen keyboard software that is easy to use and has a low number of clicks needed to input a Chinese character:

(1) Keyboard symbol classification: Classification of the keyboard symbol provides for input of the English alphabet, phonetic symbols, numeral input, special symbol input, such as punctuation marks and mathematical symbols, the Chinese phonetic alphabet, measure units, the Greek alphabet, and other specialized 
marks. This specification is based on the distinctive symbol classification of Microsoft Word.

(2) The keyboard's size can be modified. The size of a general software miniature keyboard is fixed and unchangeable, which often makes it cumbersome for a disabled person with impeded hand dexterity to enter a key, resulting in frequent typing errors. Adaptable on-screen keyboard size is a big plus for these users.

(3) Selection by temporarily hovering the mouse cursor over a key or scanning: Many users with physical disabilities are unable to mouse the mouse in a normal way. Therefore the proposed miniature keyboard software provides functions entering a key by temporarily hovering the mouse cursor over the intended key for a preset time and for selection by scanning a series of keys.

(4) Character input frequency and character editing functions: Characters can be sorted in the order of the frequency with which they were typed. Frequently entered characters are at the top of the list, thus facilitating repeated entries. These frequency lists can be custom edited by the user. The system also suggests automatic entries of commonly used character combinations.

Many different kinds of auxiliary keyboard software for disabled users are currently commercially available. Most of these only provide English key-in systems. Chinese character input with an English keyboard is extremely inconvenient for many Chinesespeaking users, therefore the assistant tool on-screen miniature keyboard that is build into the XP operating system (Fig. 5) is not a great help for them. Additionally, many commercially available Chinese input software keyboards do not provide a function for related character selection. A detailed comparison of the auxiliary software keyboards for the disabled, i.e. the assistant tool on-screen miniature keyboard built into the XP operating system, a Click-N-Type keyboard, and the proposed software, is shown in the Table I.

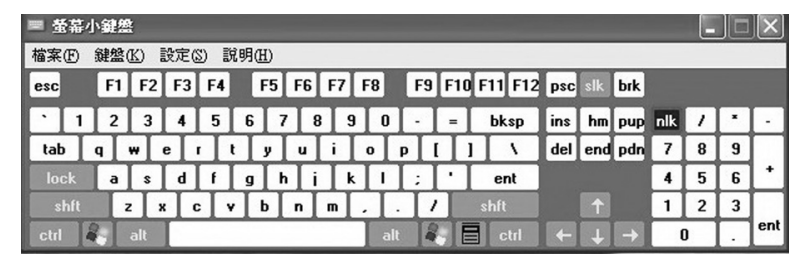

Fig. 5. The assistant tool on-screen miniature keyboard built into the XP operating system (provided solely in English).
The presented software was designed for general computer users, but also with the needs of disabled users in mind. A test was conducted in which normal users and disabled users had to use (1) the XP onscreen miniature keyboard (2) a Click-N-Type keyboard (3) this software to input Chinese characters. Ten persons were tested. Each person typed six text problems with about 100 characters. They used three kinds of on-screen keyboards: the proposed keyboard, a mini keyboard, and a Click-N-type on-screen keyboard. In table $\Pi$, the average typing time of the 10 test participants is listed for the three key-in systems. The average typing time was $12.56,14.78$, and 13.35 seconds for proposed on-screen keyboard, the mini keyboard, and Click-N-type keyboard respectively. Obviously, the proposed system is superior to the other two systems. Furthermore, the average typing speed in words per minute was $8.04,6.83$, and 7.48 for proposed on-screen keyboard, the XP on-screen miniature keyboard, and Click-N-Type keyboard respectively. The proposed system is quicker than the other two systems.

\section{CONCLUSIONS}

In this paper, an easy-to-operate on-screen virtual keyboard system for Chinese phonetic symbol input was specifically designed for people with such physical disabilities as muscle atrophy, cerebral palsy, and other severe handicaps. The proposed system provides an on-screen software keyboard, which is convenient to use, needs fewer input clicks and mouse movement, and has a high input speed. Common computer tasks like sending and receiving an email, accessing the Internet or typing and data editing can easily be achieved with it. The proposed software is certainly a good choice; Chinese and English characters can easily be entered, as well as a variety of other symbols. Various types of computer mouse devices are supported, for example a mouth control mouse, a head control mouse, or a pupil control system. This software facilitates the Chinese character input for disabled users with hand dexterity loss, thus enabling them to actively participate and contribute to today's information technology. 
Table I . Comparison of three different on-screen keyboard systems

\begin{tabular}{|c|c|c|c|}
\hline Function & $\begin{array}{c}\text { New } \\
\text { keyboard } \\
\text { system }\end{array}$ & $\begin{array}{c}\text { XP on-screen } \\
\text { miniature } \\
\text { keyboard }\end{array}$ & $\begin{array}{c}\text { Click-N-Type } \\
\text { keyboard }\end{array}$ \\
\hline $\begin{array}{c}\text { Multiple keyboard } \\
\text { types }\end{array}$ & $\checkmark$ & $x$ & $x$ \\
\hline $\begin{array}{c}\text { Choice of possible } \\
\text { phonetic combinations }\end{array}$ & $\checkmark$ & $x$ & $x$ \\
\hline $\begin{array}{c}\text { Keyboard size } \\
\text { changeable }\end{array}$ & $\checkmark$ & $x$ & $x$ \\
\hline $\begin{array}{c}\text { Keyboard mark } \\
\text { classification }\end{array}$ & $\checkmark$ & $x$ & $x$ \\
\hline $\begin{array}{c}\text { Selection by mouse } \\
\text { click }\end{array}$ & $\checkmark$ & $\checkmark$ & $\checkmark$ \\
\hline Selection by hovering & $\checkmark$ & $\checkmark$ & $\checkmark$ \\
\hline Selection by scanning & $\checkmark$ & $\checkmark$ & $\checkmark$ \\
\hline Single switch scanning & $\checkmark$ & $x$ & $x$ \\
\hline Row scanning & $\checkmark$ & $\checkmark$ & $\checkmark$ \\
\hline Forward scanning & $\checkmark$ & $\checkmark$ & $\checkmark$ \\
\hline Backward scanning & $\checkmark$ & $x$ & $x$ \\
\hline Persistent top layer & $\checkmark$ & $\checkmark$ & $\checkmark$ \\
\hline Quick tool bar & $\checkmark$ & $x$ & $x$ \\
\hline $\begin{array}{l}\text { Character frequency } \\
\text { ranking }\end{array}$ & $\checkmark$ & $x$ & $x$ \\
\hline $\begin{array}{l}\text { Character combination } \\
\text { frequency ranking }\end{array}$ & $\checkmark$ & $x$ & $x$ \\
\hline $\begin{array}{l}\text { Collects into the } \\
\text { arrangement }\end{array}$ & $\checkmark$ & $x$ & $x$ \\
\hline $\begin{array}{l}\text { Frequently edited } \\
\text { characters }\end{array}$ & $\checkmark$ & $x$ & $x$ \\
\hline User-defined terms & $\checkmark$ & $x$ & $\checkmark$ \\
\hline Macro execution & $\checkmark$ & $x$ & $\checkmark$ \\
\hline
\end{tabular}


Table II. Typing test results for three kinds of keyboard

\begin{tabular}{|l|c|c|c|c|}
\hline Problems & Number of Words & $\begin{array}{c}\text { New keyboard } \\
\text { system }\end{array}$ & $\begin{array}{c}\text { XP on-screen } \\
\text { miniature keyboard }\end{array}$ & $\begin{array}{c}\text { Click-N-Type } \\
\text { keyboard }\end{array}$ \\
\hline Text1 & 94 & 11.02 & 13.08 & 11.74 \\
\hline Text2 & 107 & 13.63 & 15.81 & 14.50 \\
\hline Text3 & 98 & 12.04 & 14.51 & 12.72 \\
\hline Text4 & 103 & 13.05 & 15.27 & 13.85 \\
\hline Text5 & 96 & 11.62 & 13.72 & 14.90 \\
\hline Text6 & 108 & 13.98 & 16.30 & 13.35 \\
\hline Average & 101 & 12.56 & 14.78 & 7.48 \\
\hline Average $(\mathrm{wpm})$ & & 8.04 & 6.83 & \\
\hline
\end{tabular}

Legends:

New: the proposed on-screen virtual keyboard system.

Mini keyboard: XP on-screen miniature keyboard.

Click-N-Type: Click-N-Type keyboard.

Wpm: words per minute

\section{REFERENCES}

1. R. Bower, et al. (Eds), The Trace ResourcebookAssistive Technology for Communication, Control, and Computer Access, Trace Research \& Development Center, Universities of WisconsinMadison, Waisman Center, 1998.

2. D.K. Anson, Alternative Computer Access: A Guide to Selection, F.A. Davis, Philadelphia, 1997.

3. T.W. King, Modern Morse Code in Rehabilitation and Education, MA: Allyn and Bacon, 2000.

4. Lloyd, L., Fuller, D., and Arvidson, H., Augmentative and alternative communication: A handbook of principles and practices, Boston: Allyn and Bacon, 1997.

5. Yang, Cheng-Hong, L.-Y. Chuang, and C.-H. Luo , Morse Code Application for Wireless Environmental Control System for Severely Disabled Individuals, IEEE Transactions on Neural Systems \& Rehabilitation Engineering, 2003; 11(4): 463-469.

6. Yang, Cheng-Hong, A Newly Developed Chinese Phonetic Morse Code for the People with Physical Impairments, Journal of Biomedical EngineeringApplications, Basis, and Communications, 1998; 10(5): 262-269.

7. Yang, Cheng-Hong, Developing a New Chinese Chang Jei Morse Code for the Disabled Persons, Chinese Journal of Medical and Biological Engineering, 1998; 18(3): 189-194.

8. Luo $\mathrm{CH}$, Shih $\mathrm{CH}$, and Shih CT, Chinese Morse code communication Auxiliary System for the Disabled, Chinese Journal of Medical and Biological Engineering, 1996; 2: 214-230.

9. Microsoft accessibility, http://www.microsoft.com/ enable/at/matvplist.aspx
10. Click-N-Type virtual keyboard, http://www.lakefolks. org/ cnt/

11. http://enable.nat.gov.tw/elearning/elearning2/ elearning2_txt.html 